\title{
THE EFFECTS OF NANO PHOSPHATIC FERTILIZER APPLICATION ON THE PRODUCTIVITY OF SOME EGYPTIAN RICE VARIETIES (ORYZA SATIVA L.)
}

\author{
Sorour, F. A. ${ }^{1}$ - Metwally, T. F..$^{2 *}$ - El-DegWy, I. S. ${ }^{1}$ - EleisAWy, E. M. -ZIDAN, A. A. ${ }^{2}$ \\ ${ }^{1}$ Agronomy Dept. Faculty of Agriculture, Kafrelsheikh University, Kafrelsheikh 33516, Egypt \\ ${ }^{2}$ Rice Res. Dept., Field Crops Res. Inst., Agric. Res. Center (ARC), Kafrelsheikh 33717, Egypt \\ *Corresponding author \\ e-mail: tamerrtc@gmail.com; phone: +20-10-9671-2828
}

(Received $31^{\text {st }}$ May 2020; accepted $14^{\text {th }}$ Sep 2020)

\begin{abstract}
A tow-season field investigation was conducted at The Experimental Farm of RRTC (Rice Research \& Training Center), Sakha, Kafr El-Sheikh Governorate, Egypt, during 2018 and 2019 to examine the impact of nano phosphatic fertilizer utilization on the performance of four Egyptian rice varieties. The selected tested varieties were Sakha106, Sakha107, Giza177, and Giza179. The phosphorus fertilizer treatments included superphosphate $\left(15 \% \mathrm{P}_{2} \mathrm{O}_{5}\right)$ as a soil application, nano-phosphorus as a foliar application, superphosphate as a foliar application and control (distilled water). Superphosphate was incorporated into the soil at the rate of $36 \mathrm{~kg} \mathrm{ha}^{-1}$ for the first treatment. Nano-phosphorus (1\%) and superphosphate (2\%) were sprayed at 35 and 45 days after transplanting (DAT) for the second and third treatments. The application of phosphorus fertilizers increased the number of tillers per $\mathrm{m}^{2}$, the number of branches per panicle, filled grains number per panicle, 1000-grain weight $(\mathrm{g})$, grain yield $\left(\mathrm{t} \mathrm{ha}^{-1}\right)$ and straw yield $\left(\mathrm{t} \mathrm{ha}^{-1}\right)$ for al tested rice varieties. Superphosphate soil application and a foliar application of nano phosphorus to Giza 179 surpassed the other plural in terms of grain and straw yields in both seasons. Application of phosphorus as superphosphate or nano-phosphorus enhanced grain quality characteristics as well as nitrogen and phosphorus content in milled grains.
\end{abstract}

Keywords: nano-phosphorus, foliar application, soil application, grain yield

\section{Introduction}

Rice (Oryza sativa L.) is an important crop for Egyptian farmers as well as for Egyptian consumers. Rice farmers in the past used a huge quantity of synthetic fertilizers to treat their fields. The synthetic fertilizers have great benefits for enhancing rice yield. On the other hand, their cost is high and their effectiveness is low. They also pollute the environment. Heavy applications of mineral fertilizers affect the groundwater and also lead to eutrophication in aquatic ecosystems. In sustainable agricultural system, rice farmers have to look for new fertilizer technologies with high efficiency and lower pollution and cost. One of those technologies is Nanoparticles' fertilizers. The impact of Nanoparticles' fertilizers on agricultural generally and rice fields especially has been reported by several investigations.

Phosphorus $(\mathrm{P})$ is critical in the metabolism of plants, playing a role in the transfer and storage of energy from photosynthesis and the metabolism of carbohydrates. It is also a structural component of the nucleic acids of genes and chromosomes and many coenzymes, phosphoproteins, and phospholipids. Application of $\mathrm{P}$ is recommended as a principal agronomical practice for increasing crop yield, given the fact that about 5.7 billion hectares globally require sufficient available P (Batjes, 1997). Phosphorus is quickly loaded inside grains of both indica and japonica rice genotypes between 6 and 15 days after flowering (Wang et al., 2016). With $\mathrm{P}$ absorption suppressed, some 
varieties can maintain a stronger ability to gain greater plant biomass and grain yield with relatively lower tissue P content (Richardson and Simpson, 2011). The yield components of rice, such as panicle number, seed-setting rate, and grain weight significantly increased with P fertilization (Usman, 2013).

Nanotechnology is a very promising field of science and technology that has the potential to open up new applications in the field of agriculture and biotechnology (Siddiqui and Al-Whaibi, 2014). Ultrafine particles are between 1 and $100 \mathrm{~nm}$ in size. Nanoparticles can serve as magic bullets containing herbicides, chemicals, or genes which target plant parts to release the content (Jampílek and Král'ová, 2015). In agriculture, fertilizers are very important for plant growth and development, most of the applied fertilizer is rendered unavailable to the plants due to many factors such as leaching, degradation by photolysis, hydrolysis and degradation. Hence it is necessary to minimize nutrient losses in fertilization and to increase the crop yield through the exploitation of new applications with the help of nanotechnology and nanomaterials. Nanoparticles have unique physicochemical properties and the potential to boost plant metabolism (Giraldo et al., 2014).

Benzon et al. (2015) reported that the application of nanotechnology in agriculture is still in its budding stage. However, it has the potential to revolutionize agricultural systems, particularly where the issues on fertilizer applications are concerned. Nano fertilizer application promoted the growth, development, and antioxidant activity in rice and has the potential to improve crop production and plant nutrition.

Upadhyaya et al. (2017) reported that calcium phosphates are of great interest in medicine, biology, agriculture, and materials sciences. They evaluated the effect of calcium phosphates nanoparticles on biochemical changes in rice. Nanoparticles increased the growth rate and affect the physiology of the plant. Calcium phosphate nanoparticles may help in the formulation of new nano growth promoters and nanofertilizers for agricultural use. Therefore, it could potentially help in the reduction of the quantity of fertilizer applied to crops and contributing to precision farming as it reduces fertilizer wastage and in turn environmental pollution due to agricultural malpractices. However, detail physiological and molecular understanding of its impact on rice crop plants is needed in the future to validate its prospective application in agriculture. This study aimed to examine the impact of nano phosphatic fertilizer utilization on the performance of four Egyptian rice varieties.

\section{Materials and methods}

At the experimental Farm of RRTC (Rice Research \& Training Center), Sakha, Kafr El-Sheikh Governorate, Egypt, a two-year field experiment was conducted during 2018 and 2019 to study the behavior of four rice cultivars treated with different sources of phosphorus fertilization.

Representative soil samples were taken from the experimental site at the depth of 0 $30 \mathrm{~cm}$ from the soil surface. Samples were air-dried, then ground to pass through a two $\mathrm{mm}$ sieve and well mixed. The procedure of soil analysis was conducted according to the methods of Black (1965) was used. The Results of chemical analysis in both seasons are shown in Table 1.

The selected tested varieties were Sakha106, Sakha107, Giza177, and Giza179 (Table 2). The treatments of phosphorus fertilizer were superphosphate $\left(15 \% \mathrm{P}_{2} \mathrm{O}_{5}\right)$ as a soil application, nano-phosphorus as a foliar application, superphosphate as a foliar 
application and control (distilled water). Superphosphate was incorporated into the soil at the rate of $36 \mathrm{~kg} \mathrm{P}_{2} \mathrm{O}_{5} \mathrm{ha}^{-1}$ for the first treatment. Nano-phosphorus (1\%) and superphosphate (2\%) were sprayed at 35 and 45 DAT for the second and third treatments.

Table 1. Soil physical and chemical properties at the experimental site in 2017 and 2018 rice growing seasons

\begin{tabular}{|c|c|c|}
\hline Soil properties & 2017 & 2018 \\
\hline \multicolumn{3}{|l|}{ Mechanical analysis: } \\
\hline Clay $\%$ & 55.04 & 54.24 \\
\hline Silt $\%$ & 30.68 & 31.56 \\
\hline Sand $\%$ & 14.28 & 14.20 \\
\hline Texture & Clay & Clay \\
\hline \multicolumn{3}{|l|}{ Chemical analysis: } \\
\hline Organic matter $\%$ & 1.48 & 1.63 \\
\hline Total nitrogen, $\mathrm{mg} \mathrm{kg}^{-1}$ & 463.08 & 469.20 \\
\hline Available $\mathrm{P}, \mathrm{mg} \mathrm{kg}^{-1}$ & 13.26 & 12.81 \\
\hline $\mathrm{pH}(1: 2.5$ soil suspension $)$ & 8.26 & 8.35 \\
\hline EC dS.m ${ }^{-1}$ (soil paste) & 1.87 & 2.14 \\
\hline \multicolumn{3}{|l|}{ Soluble cations, meq. $\mathrm{L}^{-1}$ : } \\
\hline $\mathrm{Ca}++$ & 9.72 & 10.23 \\
\hline $\mathrm{Mg}++$ & 4.05 & 4.09 \\
\hline $\mathrm{K}+$ & 1.83 & 1.87 \\
\hline $\mathrm{Na}+$ & 15.13 & 15.53 \\
\hline \multicolumn{3}{|l|}{ Soluble anions, meq. $\mathrm{L}^{-1}$ : } \\
\hline $\mathrm{CO} 3=$ & 0 & 0 \\
\hline $\mathrm{HCO} 3-$ & 6.16 & 6.93 \\
\hline $\mathrm{Cl}-$ & 8.51 & 8.65 \\
\hline $\mathrm{SO} 4=$ & 16.05 & 16.14 \\
\hline \multicolumn{3}{|l|}{ Available micronutrients ppm } \\
\hline $\mathrm{Fe}++$ & 6.22 & 5.92 \\
\hline $\mathrm{Zn}++$ & 0.72 & 0.95 \\
\hline $\mathrm{Mn}++$ & 3.57 & 3.07 \\
\hline
\end{tabular}

Phosphorus Nanoparticles (P) was obtained from rock superphosphate. According to the manufacturing and observation on Transmission Electron Microscopy (TEM), the particle size distributions were ranged from 30 to $60 \mathrm{~nm}$ (Fig. 1). Nano phosphorus solution was prepared freshly by dispersing nanoparticles in de-ionized water through ultrasonication $(300 \mathrm{~W}, 40 \mathrm{kHz})$ for $30 \mathrm{~min}$.

Table 2. Parentage, type and origin of the tested rice varieties

\begin{tabular}{c|c|c|c}
\hline Varieties & Parentage & Type & Origin \\
\hline Sakha106 & Giza 177/Hexi 30 & Japonica & Egypt \\
Sakha 107 & Giza177/BL 1 & Japonica & Egypt \\
Giza 177 & Giza 171/Yamji No.1//Pi No.4 & Japonica & Egypt \\
Giza 179 & Gz 1368-S-5-4/Gz 6296-12-1-2-1-1 & Indica/Japonica & Egypt \\
\hline
\end{tabular}




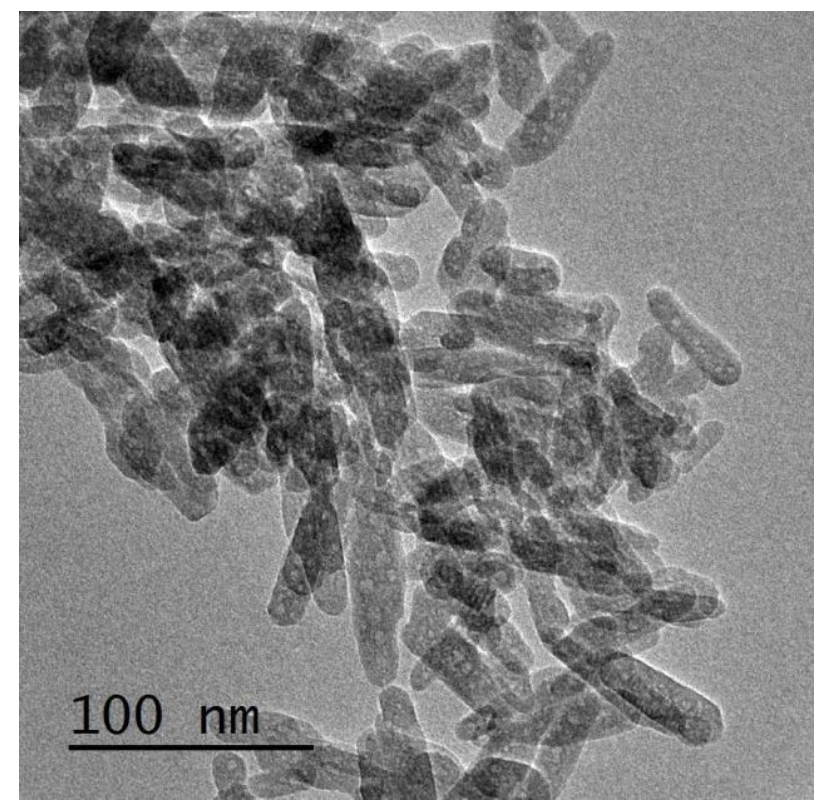

Figure 1. Phosphorus nanoparticles under the transmission electron microscopy (TEM)

Split-Plot Design with four replicates was used. Rice cultivars and phosphorus fertilization treatments were arranged as main plot and sub-plot, respectively. The plot size was $12 \mathrm{~m}^{2}$. Rice seeds were sown in the nursery on the $2^{\text {nd }}$ and $4^{\text {th }}$ of May for 2017 and, respectively. Transplanted was done after 28 days of sowing nurseries with three to four seedlings per hill, spaced 20 X $20 \mathrm{~cm}$ within and between rows. All plots received optimum cultural practices. The recommended dosages of nitrogen and potassium were applied according to the recommendations of RRTC. Nitrogen was applied at the rate of $165 \mathrm{~kg} \mathrm{~N} \mathrm{ha}^{-1}$ in the form of urea $(46 \% \mathrm{~N})$. Potassium was applied as potassium sulphate $\left(50 \% \mathrm{~K}_{2} \mathrm{O}\right)$ at the rate of $100 \mathrm{~kg} \mathrm{~K}_{2} \mathrm{O} \mathrm{ha}^{-1}$.

The studied characteristics were; number of tillers $\mathrm{m}^{-2}$, number of branches panicle ${ }^{-1}$, unfilled grains number panicle ${ }^{-1}$, filled grains number panicle ${ }^{-1}, 1000$-grain weight $(\mathrm{g})$, grain yield $\left(\mathrm{t} \mathrm{ha}^{-1}\right)$ and straw yield $\left(\mathrm{t} \mathrm{ha}^{-1}\right)$.

Grain quality characteristics: milling recovery was estimated according to Cruz and Khush (2000): 150 (g) cleaned rough rice at 14\% moisture content was dehulled using an Experimental Huller Machine (Satake - Japan). The brown rice was separated and weighed then the hulling percentage was calculated. The brown rice was milled using MC GILL Rice Miller No.2. (S.K. Appliances - India). The total milled rice was weighed and milled rice percentage was calculated. Whole milled grains were separated from the total milled rice using a rice sizing device SKU: 61-220-50 (Seedburo - USA). The percentage of head rice was calculated.

Nitrogen and phosphorus contents in milled rice grain: samples were taken from the grain after milling (50 g of milled rice). All samples were placed in paper bags and oven-dry at $70^{\circ} \mathrm{C}$ for $48 \mathrm{~h}$. Grain samples were crushed to powder and digested according to Chapman and Pratt (1961). The nitrogen content of milled grains was determined by using the Microkieldahl method (Jackson, 1967). The phosphorus content of milled grain was determined using Spectronic 1201 Spectrophotometer (Milton Roy, USA) following the procedures of Watanabe and Olsen (1965). 
The data was subjected to analysis of variance (Two-way ANOVA in randomized blocks), and the differences among treatment were compared by Duncan's Multiple Range Test $(P<0.05)$ according to Duncan $(1955)$, using Costat Statistical Software.

\section{Results and discussion}

Numbers of tillers $\mathrm{m}^{-2}$, branches panicle ${ }^{-1}$, filled grains panicle ${ }^{-1}$ and unfilled grains panicle $^{-1}$ were varied significantly among rice varieties and affected significantly by different phosphorus treatments in both seasons as shown in Table 3. Giza179 surpassed the other varieties in the above mention characteristics. The differentiation among the studied varieties in terms of those characteristics may be related to the genetic and physiological background of the tested varieties.

A foliar application of nano-phosphorus recorded the highest values of number of tillers per $\mathrm{m}^{2}$, number of branches per panicle and number of filled grains per panicle. The highest numbers of unfilled grains per panicle were recorded when phosphorus was not applied without any significant differences with the foliar application of superphosphate in the first season only. Upadhyaya et al. (2017) found that rice growth increased with an increase in the concentration of calcium phosphate nanoparticles with respect to the control. Miranda-Villagómez et al. (2019) found a positive relationship between the number of rice tillers and the initial $\mathrm{P}$ concentration in the nutrient solutions due to phosphorus application as nanoparticles.

Table 3. Tillers No. $m^{-2}$, branches No. panicle ${ }^{-1}$, filled grains panicle $e^{-1}$ and unfilled grains panicle $^{-1}$ of different rice varieties as affected by phosphorus treatment

\begin{tabular}{c|c|c|c|c|c|c|c|c}
\hline \multirow{2}{*}{ Treatments } & \multicolumn{2}{|c|}{ Tillers No. m } & \multicolumn{2}{c|}{$\begin{array}{c}\text { Branches No. } \\
\text { panicle }\end{array}$} & \multicolumn{2}{c|}{$\begin{array}{c}\text { Filled grains } \\
\text { panicle }^{-1}\end{array}$} & \multicolumn{2}{c}{$\begin{array}{c}\text { Unfilled grains } \\
\text { panicle }\end{array}$} \\
\cline { 2 - 9 } & $\mathbf{2 0 1 7}$ & $\mathbf{2 0 1 8}$ & $\mathbf{2 0 1 7}$ & $\mathbf{2 0 1 8}$ & $\mathbf{2 0 1 7}$ & $\mathbf{2 0 1 8}$ & $\mathbf{2 0 1 7}$ & $\mathbf{2 0 1 8}$ \\
\hline Rice variety: & & & & & & & & \\
Sakha 106 & $523.3 \mathrm{c}$ & $501.7 \mathrm{c}$ & $9.57 \mathrm{c}$ & $9.18 \mathrm{c}$ & $118.04 \mathrm{~d}$ & $122.8 \mathrm{c}$ & $9.88 \mathrm{~b}$ & $8.91 \mathrm{~b}$ \\
Sakha 107 & $525.1 \mathrm{c}$ & $496.6 \mathrm{c}$ & $10.00 \mathrm{~b}$ & $9.64 \mathrm{~b}$ & $125.2 \mathrm{~b}$ & $117.3 \mathrm{~b}$ & $7.59 \mathrm{~d}$ & $6.90 \mathrm{~d}$ \\
Giza 177 & $541.1 \mathrm{~b}$ & $520.0 \mathrm{~b}$ & $9.99 \mathrm{~b}$ & $9.47 \mathrm{bc}$ & $122.5 \mathrm{c}$ & $114.4 \mathrm{c}$ & $8.57 \mathrm{c}$ & $7.87 \mathrm{c}$ \\
Giza 179 & $583.1 \mathrm{a}$ & $553.6 \mathrm{a}$ & $10.50 \mathrm{a}$ & $10.40 \mathrm{a}$ & $141.8 \mathrm{a}$ & $129.8 \mathrm{a}$ & $14.08 \mathrm{a}$ & $13.25 \mathrm{a}$ \\
F test & $* *$ & $* *$ & $* *$ & $* *$ & $* *$ & $* *$ & $* *$ & $* *$ \\
\hline P treatment: & & & & & & & & \\
Super P (soil) & $584.2 \mathrm{~b}$ & $550.8 \mathrm{~b}$ & $10.37 \mathrm{~b}$ & $9.91 \mathrm{~b}$ & $134.6 \mathrm{~b}$ & $125.3 \mathrm{~b}$ & $8.01 \mathrm{~b}$ & $5.16 \mathrm{c}$ \\
Nano-P (spray) & $589.9 \mathrm{a}$ & $562.5 \mathrm{a}$ & $10.71 \mathrm{a}$ & $10.27 \mathrm{a}$ & $141.5 \mathrm{a}$ & $132.4 \mathrm{a}$ & $8.15 \mathrm{~b}$ & $6.36 \mathrm{c}$ \\
Super P (spray) & $523.7 \mathrm{c}$ & $499.1 \mathrm{c}$ & $9.91 \mathrm{c}$ & $9.43 \mathrm{c}$ & $121.8 \mathrm{~d}$ & $112.6 \mathrm{c}$ & $11.6 \mathrm{a}$ & $11.85 \mathrm{~b}$ \\
Control & $474.8 \mathrm{~d}$ & $459.6 \mathrm{~d}$ & $9.56 \mathrm{~d}$ & $9.08 \mathrm{~d}$ & $109.8 \mathrm{~d}$ & $104.01 \mathrm{~d}$ & $12.33 \mathrm{a}$ & $13.57 \mathrm{a}$ \\
F test & $* *$ & $* *$ & $* *$ & $* *$ & $* *$ & $* *$ & $* *$ & $* *$ \\
\hline Interaction effect & $* *$ & $* *$ & $* *$ & $* *$ & $* *$ & $* *$ & $* *$ & $* *$ \\
\hline
\end{tabular}

Values with the same letter in each column indicate no significant difference $(P<0.05)$

The interaction between rice variety and phosphorus treatment effect was significant for numbers of tillers $\mathrm{m}^{-2}$, branches panicle $\mathrm{e}^{-1}$, filled grains panicle $\mathrm{e}^{-1}$ and unfilled grains panicle $^{-1}$ (Table 4). A foliar application of nano-phosphorus to rice variety Giza179 recorded the highest numbers of tillers per $\mathrm{m}^{2}$, branches per panicle and filled grains per 
panicle in both seasons. There were no significant differences between the foliar application of superphosphate and nano-phosphorus to Giza179 rice variety in the first season only on number of tillers per unit area. Number of unfilled grains per panicle was higher when Giza179 did not receive any phosphorus fertilizers in both seasons which was at bar with those sprayed with superphosphate in the first season only.

Table 4. Tillers No. $m^{-2,}$ branches No. panicle ${ }^{-1}$, filled grains panicle ${ }^{-1}$ and unfilled grains panicle $^{-1}$ as affected by the interaction between rice variety and phosphorus treatment

\begin{tabular}{|c|c|c|c|c|c|c|c|c|}
\hline \multirow[b]{2}{*}{ Treatments } & \multicolumn{4}{|c|}{2017} & \multicolumn{4}{|c|}{2018} \\
\hline & $\begin{array}{c}\text { Sakha } \\
106\end{array}$ & $\begin{array}{c}\text { Sakha } \\
107\end{array}$ & $\begin{array}{c}\text { Giza } \\
177\end{array}$ & $\begin{array}{c}\text { Giza } \\
179\end{array}$ & $\begin{array}{c}\text { Sakha } \\
106\end{array}$ & $\begin{array}{c}\text { Sakha } \\
107\end{array}$ & $\begin{array}{c}\text { Giza } \\
177\end{array}$ & $\begin{array}{c}\text { Giza } \\
179\end{array}$ \\
\hline & \multicolumn{8}{|c|}{ Tillers No. $\mathrm{m}^{-2}$} \\
\hline Super P (soil) & $568.6 \mathrm{~cd}$ & $559.5 \mathrm{~d}$ & $577.1 \mathrm{bc}$ & $631.8 \mathrm{a}$ & $535.4 \mathrm{e}$ & $527.2 \mathrm{f}$ & $550.5 d$ & $590.2 \mathrm{~b}$ \\
\hline Nano-P (spray) & $578.2 \mathrm{~b}$ & $560.5 \mathrm{~d}$ & $584.5 b$ & $636.2 \mathrm{a}$ & $548.6 \mathrm{~d}$ & $535.01 \mathrm{e}$ & $559.6 \mathrm{c}$ & $606.7 \mathrm{a}$ \\
\hline Super P (spray) & $492.9 \mathrm{~h}$ & $515.7 \mathrm{f}$ & $525.9 \mathrm{e}$ & $560.3 \mathrm{~d}$ & $481.9 \mathrm{~h}$ & $470.8 \mathrm{i}$ & $511.00 \mathrm{~g}$ & $532.5 \mathrm{ef}$ \\
\hline \multirow[t]{2}{*}{ Control } & $453.6 \mathrm{k}$ & $464.6 \mathrm{j}$ & $477.1 \mathrm{i}$ & $504.02 \mathrm{~g}$ & $440.8 \mathrm{k}$ & $453.5 \mathrm{j}$ & $458.8 \mathrm{j}$ & $485.2 \mathrm{~h}$ \\
\hline & \multicolumn{8}{|c|}{ Branches No. panicle -1 $^{-1}$} \\
\hline Super P (soil) & $9.84 \mathrm{f}$ & $10.34 \mathrm{~d}$ & 10.16de & $11.13 b$ & $9.55 \mathrm{f}$ & $9.97 \mathrm{de}$ & $9.52 \mathrm{f}$ & $10.62 \mathrm{~b}$ \\
\hline Nano-P (spray) & $10.25 \mathrm{~d}$ & $10.55 \mathrm{c}$ & $10.33 \mathrm{~d}$ & $11.72 \mathrm{a}$ & $9.81 \mathrm{e}$ & $10.24 \mathrm{c}$ & $10.07 \mathrm{~cd}$ & $10.97 \mathrm{a}$ \\
\hline Super P (spray) & $9.12 \mathrm{~h}$ & $9.93 \mathrm{f}$ & $10.02 \mathrm{ef}$ & $10.58 \mathrm{c}$ & $8.85 \mathrm{~h}$ & $9.34 \mathrm{fg}$ & $9.28 \mathrm{~g}$ & $10.24 \mathrm{c}$ \\
\hline \multirow[t]{2}{*}{ Control } & $9.05 \mathrm{~h}$ & $9.42 \mathrm{~g}$ & $9.47 \mathrm{~g}$ & $10.32 d$ & $8.53 \mathrm{i}$ & $9.03 \mathrm{~h}$ & $9.00 \mathrm{~h}$ & $9.78 \mathrm{e}$ \\
\hline & \multicolumn{8}{|c|}{ Filled grains panicle $^{-1}$} \\
\hline Super P (soil) & $124.2 \mathrm{f}$ & $134.8 \mathrm{~cd}$ & $129.5 \mathrm{e}$ & $149.7 b$ & $117.6 \mathrm{~m}$ & 124.1cde & $120.7 \mathrm{ef}$ & $139.08 b$ \\
\hline Nano-P (spray) & $126.2 \mathrm{f}$ & $136.4 \mathrm{c}$ & $136.5 \mathrm{c}$ & $166.8 \mathrm{a}$ & $122.8 \mathrm{de}$ & $126.2 \mathrm{~cd}$ & $126.9 \mathrm{c}$ & $153.8 \mathrm{a}$ \\
\hline Super P (spray) & $177.8 \mathrm{~g}$ & $119.3 \mathrm{~g}$ & $117.6 \mathrm{~g}$ & $132.0 \mathrm{de}$ & $111.5 \mathrm{ij}$ & $113.9 \mathrm{hi}$ & $108.2 \mathrm{jk}$ & $116.9 \mathrm{gh}$ \\
\hline \multirow[t]{2}{*}{ Control } & $103.8 \mathrm{i}$ & $110.3 \mathrm{~h}$ & $106.4 \mathrm{i}$ & $118.7 \mathrm{~g}$ & 99.3 & 105.1 & 102.07 & 109.4 \\
\hline & \multicolumn{8}{|c|}{ Unfilled grains panicle $^{-1}$} \\
\hline Super P (soil) & $8.60 \mathrm{e}$ & $4.44 \mathrm{~g}$ & $6.48 \mathrm{f}$ & $12.52 b$ & $5.86 \mathrm{f}$ & $3.11 \mathrm{~h}$ & $3.57 \mathrm{gh}$ & $8.09 \mathrm{e}$ \\
\hline Nano-P (spray) & $10.72 \mathrm{bcd}$ & $4.80 \mathrm{fg}$ & $5.65 \mathrm{fg}$ & $11.44 \mathrm{bc}$ & $5.79 \mathrm{f}$ & $4.36 \mathrm{fgh}$ & $5 . .03 f g$ & $10.25 \mathrm{~d}$ \\
\hline Super P (spray) & $9.30 \mathrm{de}$ & $10.42 \mathrm{cde}$ & $10.39 \mathrm{cde}$ & $16.33 \mathrm{a}$ & $10.42 \mathrm{~d}$ & $10.28 \mathrm{~d}$ & $10.05 \mathrm{~d}$ & $16.67 \mathrm{~b}$ \\
\hline Control & $10.89 \mathrm{bcd}$ & $10.68 \mathrm{bcd}$ & $11.75 \mathrm{bc}$ & $16.02 \mathrm{a}$ & $13.58 \mathrm{c}$ & $9.87 \mathrm{~d}$ & $12.82 \mathrm{c}$ & $17.99 \mathrm{a}$ \\
\hline
\end{tabular}

Different letters indicate statistical significance $(P<0.05)$ within the same characteristic and the same year

1000-grain weight, grain yield and straw yield of different rice varieties were affected significantly by phosphorus treatments as well as the interaction in both seasons (Table 5). Sakha 106 and Giza 177 recorded the highest values of 1000-grain weight in the first and second seasons, respectively. The highest values of grain and straw yields were obtained by Giza179 in both seasons. Those variations among the varieties might be due to the genetic potentiality.

Application of phosphorus fertilizers as a soil or a foliar application increased significantly the values of 1000-grain weight, grain yield and straw yield over control. The heaviest 1000-grain was observed with the application of either superphosphate as a soil application or nano phosphorus as a foliar application without any significant differences between both of them. Grain and straw yields reached to the maximum values with the application of nano phosphorus as a foliar application. Wang et al. 
(2011) indicated that application of nanomaterials could increase rice grain yield. Miranda-Villagómez et al. (2019) reported that application of $\mathrm{P}$ nanoparticles is particularly important in early growth stages of rice plant. It is mobile within the plant and promotes the development of roots, tiller creation, early flowering, and maturation.

Table 5. 1000-grain weight ( $g$ ), grain yield $\left(t h a^{-1}\right)$ and straw yield $\left(t h a^{-1}\right)$ of different rice varieties as affected by phosphorus treatment

\begin{tabular}{|c|c|c|c|c|c|c|}
\hline \multirow{2}{*}{ Treatments } & \multicolumn{2}{|c|}{ 1000-grain weight $(\mathrm{g})$} & \multicolumn{2}{|c|}{ Grain yield $\left(\mathrm{t} \mathrm{ha}^{-1}\right)$} & \multicolumn{2}{|c|}{ Straw yield $\left(\mathrm{t} \mathrm{ha}^{-1}\right)$} \\
\hline & 2017 & 2018 & 2017 & 2018 & 2017 & 2018 \\
\hline Rice variety: & & & & & & \\
\hline Sakha 106 & $3.28 \mathrm{a}$ & $2.70 \mathrm{~b}$ & $10.50 \mathrm{~b}$ & $9.71 \mathrm{c}$ & $12.89 \mathrm{~b}$ & $12.78 \mathrm{c}$ \\
\hline Sakha 107 & $3.14 \mathrm{~b}$ & $2.72 \mathrm{~b}$ & $10.14 \mathrm{~d}$ & $9.41 \mathrm{~d}$ & $12.39 \mathrm{c}$ & $12.63 \mathrm{~d}$ \\
\hline Giza 177 & $3.14 \mathrm{~b}$ & $2.88 \mathrm{a}$ & $10.23 \mathrm{c}$ & $9.99 \mathrm{~b}$ & $12.81 \mathrm{~b}$ & $13.02 \mathrm{~b}$ \\
\hline Giza 179 & $3.03 \mathrm{c}$ & $2.70 \mathrm{~b}$ & $11.28 \mathrm{a}$ & $10.82 \mathrm{a}$ & $14.11 \mathrm{a}$ & $14.23 \mathrm{a}$ \\
\hline F test & $* *$ & $*$ & $* *$ & $* *$ & $* *$ & $* *$ \\
\hline P treatment: & & & & & & \\
\hline Super P (soil) & $3.30 \mathrm{a}$ & $2.86 \mathrm{a}$ & $10.97 \mathrm{ab}$ & $10.32 \mathrm{a}$ & $13.56 \mathrm{a}$ & $13.64 \mathrm{a}$ \\
\hline Nano-P (spray) & $3.30 \mathrm{a}$ & $2.88 \mathrm{a}$ & $11.02 \mathrm{a}$ & $10.40 \mathrm{a}$ & $13.56 \mathrm{a}$ & $13.71 \mathrm{a}$ \\
\hline Super P (spray) & $3.05 \mathrm{~b}$ & $2.70 \mathrm{~b}$ & $10.30 \mathrm{~b}$ & $9.75 \mathrm{~b}$ & $12.77 \mathrm{~b}$ & $12.91 \mathrm{c}$ \\
\hline Control & $2.91 \mathrm{c}$ & $2.57 \mathrm{c}$ & $9.86 \mathrm{~d}$ & $9.46 \mathrm{c}$ & $12.31 \mathrm{c}$ & $12.40 \mathrm{c}$ \\
\hline F test & $* *$ & $* *$ & $* *$ & $* *$ & $* *$ & $* *$ \\
\hline Interaction effect & $* *$ & $* *$ & $* *$ & $* *$ & $* *$ & $* *$ \\
\hline
\end{tabular}

Values with the same letter in each column indicate no significant difference $(P<0.05)$

Concerning the interaction effects on 1000-grain weight, grain yield and straw yield, data in Table 6 shows that application of phosphorus fertilizers increased 1000-grain weight for al tested rice varieties. The highest values of 1000-grain weight were recorded with the application of either superphosphate as a soil application or nano phosphorus as a foliar application to Sakha 106 in first season and to Sakha 106, Sakha 107 and Giza 177 in second season. Soil application superphosphate and a foliar application of nano phosphorus to Giza 179 surpassed the other combination in terms of grain and straw yields in both seasons.

Milling characteristics of tested rice varieties were affected significantly by phosphorus fertilizer treatments and the interaction (Table 7). Giza 179 recorded the highest percentages of hulling and milling grains. Sakha 106 recorded the highest percentage of head rice followed by Giza 177 in the first season. While Giza 177 rice variety produced the highest percentage of head rice in the second season. Milling characteristics were improved by the application of phosphorus fertilizers whatever the source and method. Application of either superphosphate as a soil application or nano phosphorus as a foliar application produced the highest percentages of hulling, milling and head rice in both seasons without any significant differences between both of them. The nano-fertilizers have a higher surface area it is mainly due to very less size of particles which provide more sites to facilitate the different metabolic processes in the plant system result production of more photosynthesis which improves the grain filling as well as milling characteristics (Qureshi et al., 2019). 


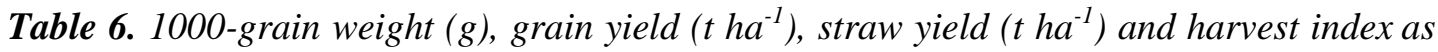
affected by the interaction between rice variety and phosphorus treatment

\begin{tabular}{c|c|c|c|c|c|c|c|c}
\hline & \multicolumn{7}{c|}{$\mathbf{2 0 1 7}$} & \multicolumn{3}{c}{$\mathbf{2 0 1 8}$} \\
\cline { 2 - 9 } Treatments & $\begin{array}{c}\text { Sakha } \\
\mathbf{1 0 6}\end{array}$ & $\begin{array}{c}\text { Sakha } \\
\mathbf{1 0 7}\end{array}$ & $\begin{array}{c}\text { Giza } \\
\mathbf{1 7 7}\end{array}$ & $\begin{array}{c}\text { Giza } \\
\mathbf{1 7 9}\end{array}$ & $\begin{array}{c}\text { Sakha } \\
\mathbf{1 0 6}\end{array}$ & $\begin{array}{c}\text { Sakha } \\
\mathbf{1 0 7}\end{array}$ & $\begin{array}{c}\text { Giza } \\
\mathbf{1 7 7}\end{array}$ & $\begin{array}{c}\text { Giza } \\
\mathbf{1 7 9}\end{array}$ \\
\hline & \multicolumn{7}{|c|}{$\mathbf{1 0 0 0 - g r a i n}$ weight (g) } \\
\hline Super P (soil) & $3.49 \mathrm{a}$ & $3.28 \mathrm{~cd}$ & $3.26 \mathrm{cde}$ & $3.17 \mathrm{def}$ & $2.86 \mathrm{a}-\mathrm{d}$ & $2.83 \mathrm{a}-\mathrm{e}$ & $2.95 \mathrm{ab}$ & $2.80 \mathrm{~b}-\mathrm{f}$ \\
Nano-P (spray) & $3.41 \mathrm{ab}$ & $3.29 \mathrm{bcd}$ & $3.33 \mathrm{bc}$ & $3.19 \mathrm{def}$ & $2.92 \mathrm{abc}$ & $2.82 \mathrm{a}-\mathrm{e}$ & $3.00 \mathrm{a}$ & $2.76 \mathrm{c}-\mathrm{g}$ \\
Super P (spray) & $3.14 \mathrm{ef}$ & $3.02 \mathrm{gh}$ & $3.08 \mathrm{fg}$ & $2.95 \mathrm{hi}$ & $2.68 \mathrm{~d}-\mathrm{g}$ & $2.63 \mathrm{fg}$ & $2.83 \mathrm{a}-\mathrm{e}$ & $2.66 \mathrm{efg}$ \\
Control & $2.94 \mathrm{hi}$ & $2.96 \mathrm{gh}$ & $2.90 \mathrm{hi}$ & $2.83 \mathrm{i}$ & $2.36 \mathrm{~h}$ & $2.60 \mathrm{~g}$ & $2.72 \mathrm{~d}-\mathrm{g}$ & $2.59 \mathrm{~g}$ \\
\hline & \multicolumn{7}{|c}{ Grain yield (t ha-1) } \\
\hline Super P (soil) & $11.06 \mathrm{~b}$ & $10.51 \mathrm{~d}$ & $10.53 \mathrm{~d}$ & $11.78 \mathrm{a}$ & $10.05 \mathrm{f}$ & $9.74 \mathrm{~h}$ & $10.26 \mathrm{~d}$ & $11.24 \mathrm{a}$ \\
Nano-P (spray) & $11.02 \mathrm{~b}$ & $10.53 \mathrm{~d}$ & $10.67 \mathrm{c}$ & $11.85 \mathrm{a}$ & $10.17 \mathrm{e}$ & $9.75 \mathrm{~h}$ & $10.38 \mathrm{c}$ & $11.29 \mathrm{a}$ \\
Super P (spray) & $10.24 \mathrm{e}$ & $9.85 \mathrm{f}$ & $10.17 \mathrm{e}$ & $10.95 \mathrm{~b}$ & $9.50 \mathrm{i}$ & $9.17 \mathrm{i}$ & $9.84 \mathrm{~g}$ & $10.51 \mathrm{~b}$ \\
Control & $9.66 \mathrm{~g}$ & $9.67 \mathrm{~g}$ & $9.56 \mathrm{~g}$ & $10.53 \mathrm{~d}$ & $9.13 \mathrm{i}$ & $8.99 \mathrm{k}$ & $9.47 \mathrm{i}$ & $10.25 \mathrm{~d}$ \\
\hline & \multicolumn{7}{|c}{ Straw yield (t ha-1) } \\
\hline Super P (soil) & $13.39 \mathrm{c}$ & $12.82 \mathrm{e}$ & $13.25 \mathrm{~cd}$ & $14.76 \mathrm{a}$ & $13.30 \mathrm{~d}$ & $13.10 \mathrm{e}$ & $13.35 \mathrm{~d}$ & $14.81 \mathrm{a}$ \\
Nano-P (spray) & $13.68 \mathrm{~b}$ & $12.4 \mathrm{~g}$ & $13.27 \mathrm{~cd}$ & $14.82 \mathrm{a}$ & $13.41 \mathrm{~cd}$ & $13.05 \mathrm{ef}$ & $13.5 \mathrm{c}$ & $14.90 \mathrm{a}$ \\
Super P (spray) & $12.53 \mathrm{fg}$ & $12.2 \mathrm{~h}$ & $12.68 \mathrm{ef}$ & $13.64 \mathrm{~b}$ & $12.45 \mathrm{~g}$ & $12.31 \mathrm{~h}$ & $12.95 \mathrm{f}$ & $13.93 \mathrm{~b}$ \\
Control & $11.95 \mathrm{i}$ & $12.02 \mathrm{i}$ & $12.05 \mathrm{i}$ & $13.22 \mathrm{~d}$ & $11.97 \mathrm{i}$ & $12.06 \mathrm{i}$ & $12.27 \mathrm{~h}$ & $13.30 \mathrm{~d}$ \\
\hline
\end{tabular}

Different letters indicate statistical significance $(P<0.05)$ within the same characteristic and the same year

Table 7. Hulling, milling and head rice \% of different rice varieties as affected by phosphorus treatment

\begin{tabular}{c|c|c|c|c|c|c}
\hline \multirow{2}{*}{ Treatments } & \multicolumn{2}{|c|}{ Hulling \% } & \multicolumn{2}{c|}{ Milling \% } & \multicolumn{2}{c}{ Head rice \% } \\
\cline { 2 - 7 } & $\mathbf{2 0 1 7}$ & $\mathbf{2 0 1 8}$ & $\mathbf{2 0 1 7}$ & $\mathbf{2 0 1 8}$ & $\mathbf{2 0 1 7}$ & $\mathbf{2 0 1 8}$ \\
\hline Rice variety: & & & & & & \\
Sakha 106 & $76.21 \mathrm{~b}$ & $75.26 \mathrm{c}$ & $68.48 \mathrm{~b}$ & $67.06 \mathrm{c}$ & $65.64 \mathrm{a}$ & $60.28 \mathrm{~b}$ \\
Sakha 107 & $73.27 \mathrm{c}$ & $74.06 \mathrm{~d}$ & $65.14 \mathrm{~d}$ & $66.42 \mathrm{c}$ & $60.39 \mathrm{c}$ & $59.32 \mathrm{c}$ \\
Giza 177 & $73.74 \mathrm{c}$ & $78.29 \mathrm{~b}$ & $66.92 \mathrm{c}$ & $69.40 \mathrm{~b}$ & $64.17 \mathrm{~b}$ & $63.36 \mathrm{a}$ \\
Giza 179 & $78.50 \mathrm{a}$ & $81.33 \mathrm{a}$ & $71.44 \mathrm{a}$ & $72.68 \mathrm{a}$ & $59.82 \mathrm{~d}$ & $60.32 \mathrm{~b}$ \\
F test & $* *$ & $* *$ & $* *$ & $* *$ & $* *$ & $* *$ \\
\hline P treatment: & & & & & & \\
Super P (soil) & $78.77 \mathrm{a}$ & $79.66 \mathrm{a}$ & $70.47 \mathrm{a}$ & $70.78 \mathrm{a}$ & $67.18 \mathrm{a}$ & $63.67 \mathrm{~b}$ \\
Nano-P (spray) & $78.92 \mathrm{a}$ & $80.10 \mathrm{a}$ & $70.80 \mathrm{a}$ & $71.19 \mathrm{a}$ & $66.73 \mathrm{a}$ & $64.60 \mathrm{a}$ \\
Super P (spray) & $72.67 \mathrm{~b}$ & $75.23 \mathrm{~b}$ & $65.92 \mathrm{~b}$ & $67.43 \mathrm{~b}$ & $59.57 \mathrm{~b}$ & $58.27 \mathrm{c}$ \\
Control & $71.38 \mathrm{c}$ & $73.95 \mathrm{c}$ & $64.78 \mathrm{c}$ & $66.17 \mathrm{c}$ & $56.55 \mathrm{c}$ & $56.73 \mathrm{~d}$ \\
F test & $* *$ & $* *$ & $* *$ & $* *$ & $* *$ & $* *$ \\
\hline Interaction effect & $* *$ & $* *$ & $* *$ & $* *$ & $* *$ & $* *$ \\
\hline
\end{tabular}

Values with the same letter in each column indicate no significant difference $(P<0.05)$

Regarding the interaction effect, the application of phosphorus fertilizers enhanced them milling characteristics of all tested varieties (Table 8). The highest percentage of hulling was obtained when superphosphate as a soil application or nano phosphorus as a 
foliar application was applied to Sakha 106 and Giza 179 in first season and to Giza 179 only in the second season. Application of superphosphate as a soil application or nano phosphorus as a foliar application to Giza 179 recorded the highest values of milling percentage in both seasons. Moreover, there were no significant differences between the effect of mentioned treatments on milling percentage and application of superphosphate as a soil application in first season only. The highest percentages of head rice were observed when superphosphate as a soil application or nano phosphorus as a foliar application was applied to Sakha 106 and Giza 177 in the first and second season respectively.

Table 8. Hulling, milling and head rice \% as affected by the interaction between rice variety and phosphorus treatment

\begin{tabular}{|c|c|c|c|c|c|c|c|c|}
\hline \multirow[b]{2}{*}{ Treatments } & \multicolumn{4}{|c|}{2017} & \multicolumn{4}{|c|}{2018} \\
\hline & $\begin{array}{c}\text { Sakha } \\
106\end{array}$ & $\begin{array}{c}\text { Sakha } \\
107\end{array}$ & $\begin{array}{c}\text { Giza } \\
177\end{array}$ & $\begin{array}{c}\text { Giza } \\
179\end{array}$ & $\begin{array}{c}\text { Sakha } \\
106\end{array}$ & $\begin{array}{c}\text { Sakha } \\
107\end{array}$ & $\begin{array}{c}\text { Giza } \\
177\end{array}$ & $\begin{array}{c}\text { Giza } \\
179\end{array}$ \\
\hline & \multicolumn{8}{|c|}{ Hulling \% } \\
\hline Super P (soil) & $81.41 \mathrm{a}$ & $76.68 \mathrm{~cd}$ & $76.08 \mathrm{~cd}$ & $80.92 \mathrm{a}$ & $77.80 \mathrm{e}$ & $77.09 \mathrm{e}$ & $80.42 \mathrm{~cd}$ & $83.35 \mathrm{a}$ \\
\hline Nano-P (spray) & $79.63 \mathrm{ab}$ & $76.78 \mathrm{~cd}$ & $77.84 \mathrm{bc}$ & $81.42 \mathrm{a}$ & $79.50 \mathrm{~d}$ & $76.89 \mathrm{e}$ & $81.70 \mathrm{bc}$ & $82.34 \mathrm{ab}$ \\
\hline Super P (spray) & $73.33 \mathrm{ef}$ & $70.44 \mathrm{gh}$ & $72.04 \mathrm{fg}$ & $74.85 \mathrm{de}$ & $73.06 f$ & $71.55 \mathrm{~g}$ & $77.11 \mathrm{e}$ & $79.20 \mathrm{~d}$ \\
\hline \multirow[t]{2}{*}{ Control } & $70.49 \mathrm{gh}$ & $69.20 \mathrm{~h}$ & $69.01 \mathrm{~h}$ & $76.82 \mathrm{c}$ & $70.67 \mathrm{~g}$ & $70.74 \mathrm{~g}$ & $73.94 \mathrm{f}$ & $80.46 \mathrm{~cd}$ \\
\hline & \multicolumn{8}{|c|}{ Milling \% } \\
\hline Super P (soil) & $72.74 \mathrm{ab}$ & $67.43 \mathrm{ef}$ & $68.72 \mathrm{de}$ & $73.01 \mathrm{ab}$ & $69.21 \mathrm{~d}$ & $68.81 \mathrm{~d}$ & $70.89 b c$ & $74.19 \mathrm{a}$ \\
\hline Nano-P (spray) & $71.53 \mathrm{bc}$ & $67.68 \mathrm{e}$ & $70.12 \mathrm{~cd}$ & $73.88 \mathrm{a}$ & $70.46 \mathrm{c}$ & $68.71 \mathrm{~d}$ & $71.84 \mathrm{~b}$ & $73.75 \mathrm{a}$ \\
\hline Super P (spray) & $65.70 \mathrm{~g}$ & $62.80 \mathrm{~h}$ & $65.98 \mathrm{fg}$ & $69.22 \mathrm{~d}$ & $64.76 f g$ & $64.92 \mathrm{f}$ & $68.6 \mathrm{~d}$ & $71.46 \mathrm{~b}$ \\
\hline \multirow[t]{2}{*}{ Control } & $63.97 \mathrm{~h}$ & $62.63 \mathrm{~h}$ & $62.87 \mathrm{~h}$ & $69.65 \mathrm{~d}$ & $63.83 \mathrm{gh}$ & $63.23 \mathrm{~h}$ & $66.29 \mathrm{e}$ & $71.33 \mathrm{bc}$ \\
\hline & \multicolumn{8}{|c|}{ Head rice \% } \\
\hline Super P (soil) & $72.05 \mathrm{a}$ & $64.95 \mathrm{cde}$ & $64.82 \mathrm{cde}$ & $66.88 b c$ & $64.07 \mathrm{bc}$ & $61.85 \mathrm{~d}$ & $65.39 \mathrm{ab}$ & $63.38 \mathrm{~cd}$ \\
\hline Nano-P (spray) & $70.62 \mathrm{a}$ & $62.85 \mathrm{e}$ & $67.61 \mathrm{~b}$ & $65.83 \mathrm{bcd}$ & $65.05 \mathrm{bc}$ & $61.94 \mathrm{~d}$ & $66.93 a$ & $64.49 \mathrm{bc}$ \\
\hline Super P (spray) & $60.28 f$ & $58.66 \mathrm{f}$ & $64.25 \mathrm{de}$ & $55.08 \mathrm{~g}$ & $55.88 \mathrm{gh}$ & $57.67 \mathrm{ef}$ & $61.91 d$ & $57.63 \mathrm{efg}$ \\
\hline Control & $59.61 \mathrm{f}$ & $55.08 \mathrm{~g}$ & $60.00 \mathrm{f}$ & $51.49 \mathrm{~h}$ & $56.11 \mathrm{fgh}$ & $55.82 \mathrm{~h}$ & $59.20 \mathrm{e}$ & $55.79 \mathrm{~h}$ \\
\hline
\end{tabular}

Different letters indicate statistical significance $(P<0.05)$ within the same characteristic and the same year

The results of post-harvest milled grain analysis indicated that there were changes in nitrogen and phosphorus content percentages among the tested rice varieties as well as among phosphorus treatments (Table 9). Data showed that the highest values of nitrogen and phosphorus content percentages were obtained in Giza 179 rice variety. On the other hand application of phosphorus fertilizers as a soil or a foliar application enhanced the absorption of nitrogen and phosphorus by rice grains. Regarding the interaction effect, data in Table 10 showed that the application of different phosphorus treatments increased the uptake of nitrogen and phosphorus by all rice varieties. These results confirm that nutrients uptake is controlled by source and method of phosphorus application which was observed also by Miranda-Villagómez et al. (2019). Rock phosphate if use as nano form it may increase the availability of phosphorus to the plant because the direct application of rock phosphate nanoparticles on the crop may prevent 
fixation in the soil similarly there is no silicic acid, iron, and calcium for fixation of the phosphorus hence it increases phosphorus availability to the crop plants (Qureshi et al., 2019; Suriyaprabha et al., 2012). Wang et al., 2011 found that applying nanomaterials fertilizer can increase NPK absorption amount of rice plants and promoting transportation of those nutrients to grains. Dhansil et al., 2018 observed that the application of phosphorus nanoparticles showed a positive relationship between nitrogen and phosphorus uptake by pearl millet.

Table 9. Nitrogen (\%) and Phosphorus (\%) of the tested rice varieties under phosphorus fertilizer treatments

\begin{tabular}{c|c|c|c|c}
\hline \multirow{2}{*}{ Treatment } & \multicolumn{2}{|c|}{ Nitrogen (\%) } & \multicolumn{2}{c}{ Phosphorus (\%) } \\
\cline { 2 - 5 } & $\mathbf{2 0 1 7}$ & $\mathbf{2 0 1 8}$ & $\mathbf{2 0 1 7}$ & $\mathbf{2 0 1 8}$ \\
\hline Varieties: & & & & \\
Sakha 106 & $1.15 \mathrm{~b}$ & $1.19 \mathrm{~b}$ & $0.400 \mathrm{~b}$ & $0.455 \mathrm{~b}$ \\
Sakha 107 & $1.16 \mathrm{~b}$ & $1.16 \mathrm{c}$ & $0.39 \mathrm{~b}$ & $0.42 \mathrm{c}$ \\
Giza 177 & $1.08 \mathrm{c}$ & $1.10 \mathrm{~d}$ & $0.39 \mathrm{~b}$ & $0.42 \mathrm{c}$ \\
Giza 179 & $1.24 \mathrm{a}$ & $1.30 \mathrm{a}$ & $0.43 \mathrm{a}$ & $0.48 \mathrm{a}$ \\
F test & $* *$ & $* *$ & $* *$ & $* *$ \\
P treatments: & & & & $0.46 \mathrm{~b}$ \\
Super P (soil) & $1.19 \mathrm{~b}$ & $1.25 \mathrm{~b}$ & $0.41 \mathrm{a}$ & $0.48 \mathrm{a}$ \\
Nano-P (spray) & $1.23 \mathrm{a}$ & $1.29 \mathrm{a}$ & $0.42 \mathrm{a}$ & $0.47 \mathrm{ab}$ \\
Super P (spray) & $1.20 \mathrm{ab}$ & $1.20 \mathrm{c}$ & $0.41 \mathrm{a}$ & $0.40 \mathrm{c}$ \\
Control & $1.02 \mathrm{c}$ & $1.02 \mathrm{~d}$ & $0.37 \mathrm{~b}$ & $* *$ \\
\hline F test & $* *$ & $* *$ & $* *$ & $* *$ \\
\hline Interaction effect & $* *$ & $* *$ & $* *$ & \\
\hline
\end{tabular}

Values with the same letter in each column indicate no significant difference $(P<0.05)$

Table 10. Nitrogen (\%) and phosphorus (\%) contents in milled rice grains as affected by the interaction between rice variety and phosphorus treatment

\begin{tabular}{c|c|c|c|c|c|c|c|c|c}
\hline \multirow{2}{*}{ Treatments } & \multicolumn{7}{c}{$\mathbf{2 0 1 7}$} & \multicolumn{5}{c}{$\mathbf{2 0 1 8}$} \\
\cline { 2 - 9 } & $\begin{array}{c}\text { Sakha } \\
\mathbf{1 0 6}\end{array}$ & $\begin{array}{c}\text { Sakha } \\
\mathbf{1 0 7}\end{array}$ & $\begin{array}{c}\text { Giza } \\
\mathbf{1 7 7}\end{array}$ & $\begin{array}{c}\text { Giza } \\
\mathbf{1 7 9}\end{array}$ & $\begin{array}{c}\text { Sakha } \\
\mathbf{1 0 6}\end{array}$ & $\begin{array}{c}\text { Sakha } \\
\mathbf{1 0 7}\end{array}$ & $\begin{array}{c}\text { Giza } \\
\mathbf{1 7 7}\end{array}$ & $\begin{array}{c}\text { Giza } \\
\mathbf{1 7 9}\end{array}$ \\
\hline & \multicolumn{7}{|c|}{ Nitrogen $(\mathbf{\%})$} \\
\hline Super P (soil) & $1.20 \mathrm{cde}$ & $1.19 \mathrm{def}$ & $1.08 \mathrm{gh}$ & $1 . .28 \mathrm{ab}$ & $1.27 \mathrm{~cd}$ & $1.22 \mathrm{de}$ & $1.12 \mathrm{f}$ & $1 . .39 \mathrm{~b}$ \\
Nano-P (spray) & $1.22 \mathrm{bcd}$ & $1.26 \mathrm{abc}$ & $1.12 \mathrm{fgh}$ & $1.30 \mathrm{a}$ & $1.20 \mathrm{e}$ & $1.28 \mathrm{c}$ & $1.13 \mathrm{f}$ & $1.52 \mathrm{a}$ \\
Super P (spray) & $1.20 \mathrm{~cd}$ & $1.13 \mathrm{efg}$ & $1.18 \mathrm{def}$ & $1.30 \mathrm{a}$ & $1.27 \mathrm{~cd}$ & $1.13 \mathrm{f}$ & $1.12 \mathrm{f}$ & $1.28 \mathrm{c}$ \\
Control & $1.00 \mathrm{ij}$ & $1.05 \mathrm{hi}$ & $0.95 \mathrm{j}$ & $1.07 \mathrm{gh}$ & $1.02 \mathrm{~g}$ & $1.02 \mathrm{~g}$ & $1.04 \mathrm{~g}$ & $1.02 \mathrm{~g}$ \\
\hline & \multicolumn{7}{|c|}{ Phosphorus $(\mathbf{\%})$} \\
Super P (soil) & $0.41 \mathrm{bc}$ & $0.40 \mathrm{bcd}$ & $0.36 \mathrm{ef}$ & $0.46 \mathrm{a}$ & $0.43 \mathrm{def}$ & $0.47 \mathrm{bc}$ & $0.44 \mathrm{cde}$ & $0.49 \mathrm{~b}$ \\
Nano-P (spray) & $0.41 \mathrm{bc}$ & $0.41 \mathrm{bc}$ & $0.41 \mathrm{bcd}$ & $0.43 \mathrm{~b}$ & $0.49 \mathrm{~b}$ & $0.48 \mathrm{~b}$ & $0.40 \mathrm{fg}$ & $0.54 \mathrm{a}$ \\
Super P (spray) & $0.42 \mathrm{bc}$ & $0.38 \mathrm{de}$ & $0.39 \mathrm{cde}$ & $0.46 \mathrm{a}$ & $0.50 \mathrm{ab}$ & $0.45 \mathrm{~cd}$ & $0.43 \mathrm{def}$ & $0.49 \mathrm{~b}$ \\
Control & $0.35 \mathrm{f}$ & $0.36 \mathrm{ef}$ & $0.42 \mathrm{~b}$ & $0.36 \mathrm{ef}$ & $0.38 \mathrm{~g}$ & $0.40 \mathrm{fg}$ & $0.41 \mathrm{ef}$ & $0.40 \mathrm{fg}$ \\
\hline
\end{tabular}

Different letters indicate statistical significance $(P<0.05)$ within the same characteristic and the same year 


\section{Conclusion}

The results demonstrated that the tested rice varieties displayed differential phosphorus response depending on the source and method of phosphorus fertilizer used. Phosphorus supplied from superphosphate as a soil application exhibited a higher response than supplied as foliar application. Nano-phosphorus application as a foliar promoted yield, quality and NP uptake by rice grain. Furthers studies are needed on the effect of nano-phosphorus on rice varieties behavior under Egyptian condition.

\section{REFERENCES}

[1] Batjes, N. H. (1997): A world data set of derived soil properties by FAO-UNESCO soil unit for global modeling. - Soil Use and Management 13(1): 9-16.

[2] Benzon, H. R. L., Rubenecia, M. R. U., Ultra Jr, V. U., Lee, S. C. (2015): Nano-fertilizer affects the growth, development, and chemical properties of rice. - International Journal of Agronomy and Agricultural Research 7(1): 105-117.

[3] Black, C. A. (1965): Methods of Soil Analysis. Part 2: Chemical and Microbial Properties. - Number 9 in Series Agronomy. American Society of Agronomy, Inc., Madison, WI.

[4] Chapman, H. D., Pratt, P. F. (1961): Method of Analysis for Soils Plant and Water. California Citrus Experiment Station, Univ. of California, Division of Agricultural Sciences, USA.

[5] Cruz, N. D., Khush, G. S. (2000): Rice Grain Quality Evaluation Procedures. - In: Singh, R. K. et al. (eds.) Aromatic Rices. Mohan Primlani for Oxford \& IBH Publishing, New Delhi, pp. 15-28.

[6] Dhansil, A., Zalawadia, N. M., Prajapat, B. S., Yadav, K. (2018): Effect of nano phosphatic fertilizer on nutrient content and uptake by pearl millet (Pennisetum glaucum L.) crop. - International Journal of Current Microbiology and Applied Sciences 7(12): 2327-2337.

[7] Duncan, B. (1955): Multiple range and multiple F. test. - Biometrics 11: 1-42.

[8] Miranda-Villagómez, E., Trejo-Téllez, L. I., Gómez-Merino, F. C., Sandoval-Villa, M., Sánchez-García, P., Aguilar-Méndez, M. Á. (2019): Nanophosphorus fertilizer stimulates growth and photosynthetic activity and improves $\mathrm{P}$ status in rice. - Journal of Nanomaterials. https://doi.org/10.1155/2019/5368027.

[9] Giraldo, J. P., Landry, M. P., Faltermeier, S. M., McNicholas, T. P., Iverson, N. M., Boghossian, A. A., Reuel, N. F., Hilmer, A. J., Sen, F., Brew, J. A., Strano, M. S. (2014): Plant nanobionics approach to augment photosynthesis and biochemical sensing. - Nature Materials 13: 400-408.

[10] Jackson, M. L. (1967): Soil Chemical Analysis. - Prentice Hall of India, New Delhi, pp. 144-197.

[11] Jampílek, J., Králová, K. (2015): Application of nanotechnology in agriculture and food industry, its prospects and risks. - Ecological Chemistry and Engineering Science 22(3): 321-361.

[12] Suriyaprabha, R., Karunakaran, G., Yuvakkumar, R., Rajendran, V., Kannan, N. (2012): Silica nanoparticles for increased silica availability in maize (Zea mays. L) seeds under hydroponic conditions. - Current Nanoscience 8(6): 902-908.

[13] Qureshi, A., Singh, D. K., Dwivedi, S. (2019): Nano-fertilizers: a novel way for enhancing nutrient use efficiency and crop productivity. - International Journal of Current Microbiology and Applied Sciences 7(2): 3325-3335.

[14] Richardson, A. E., Simpson, R. J. (2011): Soil microorganisms mediating phosphorus availability. - Plant Physiology 156(3): 989-996. 
[15] Siddiqui, M. H., Al-Whaibi, M. H. (2014): Role of nano- $\mathrm{SiO}_{2}$ in germination of tomato (Lycopersicum esculentum, seeds Mill.). - Saudi Journal of Biological Sciences 21(1): 13-17.

[16] Upadhyaya, H., Begum, L., Dey, B., Nath, P. K., Panda, S. K. (2017): Impact of calcium phosphate nanoparticles on rice plant. - Journal of Plant Science and Phytopathology 1: $1-10$.

[17] Usman, K. (2013): Effect of phosphorus and irrigation levels on yield, water productivity, phosphorus use efficiency and income of lowland rice in northwest Pakistan. - Rice Science 20(1): 61-72.

[18] Wang, F., Rose, T., Jeong, K., Kretzschmar, T., Wissuwa, M. (2016): The knowns and unknowns of phosphorus loading into grains, and implications for phosphorus efficiency in cropping systems. - Journal of Experimental Botany 67(5): 1221-1229.

[19] Wang, X. J., Song, H. X., Liu, Q., Rong, X. M., Peng, J. W., Xie, G. X., Zhang, Z. H., Wang, S. J. (2011): Effects of nanopreparation coated nitrogen fertilizer on nutrient absorption and yield of early rice. - Hunan Agricultural Sciences 11: 021.

[20] Watanabe, F. S., Olsen, S. R. (1965): Test of an ascorbic acid method for determining phosphorus in water and $\mathrm{NaHCO}_{3}$ extracts from soils. - Soil Science Society of America Journal S29(6): 677-678. 